\title{
Acute Ischemic Stroke and Acute on Chronic Kidney Disease
}

\author{
Raja Ahsan Aftab, ${ }^{1}$ Amer Hayat Khan, ${ }^{1}$ Hadeer Akram AbdulRaazaq, ${ }^{1}$ Azreen Syazril Adnan' \\ 'Department of Clinical Pharmacy, School of Pharmaceutical Sciences, Universiti Sains Malaysia, Malaysia.
}

\begin{abstract}
Ischemic stroke is due to either local thrombus formation or emboli that occlude a cerebral artery, together with chronic kidney disease represent major mortality and morbidity. Here wer present a case of 53 years old Malay man, admitted to a hospital in Malaysia complaining of sudden onset of weakness on right sided upper and lower limb associated with slurred speech. Patient was also suffering from uncontrolled hypertension, hyperlipidemia, chronic kidney disease stage 4, and diabetes mellitus(un controlled). He was diagnosed with acute ischemic stroke with cranial nerve 7 palsy (with right hemiparesis), acute on chronic kidney disease precipitated by dehydration and ACE inhibitor, and hyperkalemia. Patients with ischemic disease and chronic kidney disaese require constant monitering and carefull selected pharmacotherapy. Patient was placed under observation and was prescribed multiple pharamacotherpay to stabalise detoriating condition
\end{abstract}

Keywords: ischemic disease; chronic kidney disease; uncontrolled hypertension.

\section{INTRODUCTION}

Cerebral artherosclerosis is a causative factor in most cases of ischemic stroke, although $30 \%$ are of unknown etiology. In carotid artherosclerosis plaques may rupture, resulting in collagen exposure, platelet aggregation, and thrombus formation. The clot may cause local occlusion or may dislodge and travel distally, eventually occluding a cerebral vessel. ${ }^{1}$ The patient may experience weakness on one side of the body, inability to speak, loss of vision, vertigo, or falling.

Chronic kidney disease (CKD) is a progressive loss of function over several months to years, characterized by gradual replacement of normal kidney architecture with interstitial fibrosi. ${ }^{2}$ CKD is characterized by the level of kidney function, based on glomerular filtration rate (GFR) into stages 1 to 5 , with each increasing number indicating a more advanced stage of the disease, as defined by a declining GFR.

\section{CASE REPORT}

A 53 years old Malay man, admitted to Hospital Universiti Sains Malaysia (HUSM) complaining of sudden onset of weakness on right sided upper and lower limb associated with slurred speech . patient claimed that there is no chest pain and shortness of breath. He also complained off poor orl intake for 2 weeks already and has on and off vomiting. patient urine output was still good, care taker reported that patient was on his way for massage where he complained of right limb weakness and became unbalanced, along with slurred speech, chest pain and assymetrical of the face. The patient was treated for stroke at a loccal clinic and was then shifted to HUSM.

Correspondence: Raja Ahsan Aftab, Department of Clinical Pharmacy, School of Pharmaceutical Sciences, Universiti Sains Malaysia, Malaysia. Email: ahsan1025@yahoo.com, Phone: +60104322719. 
Patient is currently suffering from uncontrolled hypertension, hyperlipidemia, chronic kidney disease stage 4, and diabetes mellitus(un controlled). Subject has been suffering from diabetes mellitus since 11 years. In 2010, patient had a stroke but recovers well. Subject claimed to be suffering from hypertension at age of 30 but was not on any medication and only start taking anti-hypertensive in 2003. Patient was diagnosed with acute ischemic stroke with cranial nerve 7 palsy (with right hemiparesis), acute on chronic kidney disease precipitated by dehydration and ACE inhibitor, and hyperkalemia.

On examination they found positive loss of nasolabial fold. Neurological examination showed that cranial nerve 7 is impaired (lower motor neuron weakness and decrease in sensation). The impaired of cranial nerve 7 cause loss in the control of muscles of facial expression. Cranial nerves 9 and 10 are not impaired, thus his gag reflex is present. CT scan showed no intra cranial bleeding(ICB) and hypodense lession at middle cerebral artery (MCA).

\section{DISCUSSION}

ACE inhibitors decrease aldosterone and can increase serum potassium concentrations. Hyperkalemia occurs primarily in patients with chronic kidney disease, or diabetes mellitus, and in those also taking ARBs, NSAIDs, potassium supplements, or potassium-sparing diuretics. ${ }^{3}$ Current patient is suffering from chronic kidney disease and diabtes mellitus, and is taking ACE inhibitors for his hypertension. ACE inhibitors worsens current patient condition, and increase in serum potassium concentrations $(5.8 \mathrm{mmol} / \mathrm{L})$. This precipitates patient chronic kidney disease, because it is widely known that chronic kidney disease can cause imbalance fluid and electrolyte in the body, and apart from that, dehydration worsen the patient condition. ${ }^{4}$ Glomerular filtration rate (GFR) decrease in patients receiving ACE inhibitors because of inhibition of angiotensin 2 vasoconstriction on effecrent arterioles, and caused elevation in serum creatinine concentration $(256 \mu \mathrm{mol} / \mathrm{L})$. Thus, perindopril(ACE inhibitor) is withhold in current patient. To improve patient hydration status, doctor gave him with IV drip of dextrose and normal saline for 24 hourly.

patient was given cocktail infusion that included glucose, insulin and calcium gluconate, this helps to shift the potassium back into the cell, thus reduce the level of potassium in blood. Patient was also prescribed with oral kalamate $15 \mathrm{mg}$ TDS since it is a calcium polystyrene sulfonate powder used as serum potassium lowering agent. Patient potassium level improved after the treatment $(3.9 \mathrm{mmol} / \mathrm{L})$.

Patient is diagnosed with acute ischemic stroke (left cerebrovascularaccident with right hemiparesis). The goal of therapy in current patient is to reduce the ongoing neurologic injury and decrease mortality and long term disability, prevent complication secondary to immobility and neuroogic dysfunction, and lastly prevent stroke recurrence. Based on the Stroke Council of The American Stroke Association, ${ }^{5}$ the drug treatment of first choice for acute ischemic stroke is IV Alteplase (t-PA) $0.9 \mathrm{mg} / \mathrm{kg}$ (maximum $90 \mathrm{mg}$ ), with $10 \%$ of the dose given as a bolus over 1 minute and the remainder infused over 60 minutes. It must be given within 4.5 hours of stroke symptom onset. Early reperfusion with t-PA can significantly reduced the ultimate disability caused by ischemic stroke. Other alternative includes aspirin $325 \mathrm{mg}$ to be administered within $24 \mathrm{hrs}$ to $48 \mathrm{hrs}$ of the onset of stroke symptoms. Early aspirin therapy reduces the risk of early stroke recurrence and long term death and disability. If tPA is used, aspirin should not be given until 24-48 hours after the tPA infusion. ${ }^{6}$ Patient was prescribed with table aspirin 150mg OD starting. Low effective dose of aspirin is given to avoid any complication of gastrointestinal bleeding.

Patient is also suffering from uncontrolled hypertension and diabetes mellitus type 2. Patient is prescribed tablet felodipine $10 \mathrm{mg}$ OD to control his hypertension along with tablet furosemide $40 \mathrm{mg}$ OD. Furosemide is the suitable diuretic agent to be used as antihypertensive agent as patient creatinine clearance is $<30 \mathrm{ml} / \mathrm{min}$. Carvedilol is a good choice of beta blocker as it can reduce cardiovascular risk. Subcutaneous Actrapid 8 unit TDS is presribed for his diabetes mellitus. Diabetes mellitus and hypertension can worsen patient chronic kidney disease, as uncontrolled diabetes mellitus can cause nephropathy and proteinuria.

Since patient is also diagnosed hyperlipidemia, he was prescribed with tablet atorvastatin $20 \mathrm{mg}$ ON with tablet ezetimibe 10mg OD . Lastly, subject was on tablet pantoprazole 40mg OD to reduce gastric acid secretion. It is used as prophylaxis treatment in patient, to avoid stress induced ulcer.

Hypertensive patients with chronic kidney disaese represnts a challange to doctors in slecting an appropiate pharmacotherapy. Monitering of renal function and reational prescribing can aid in reducing mortality and morbidty in patients with hypertension and chronic kidney disase. 


\section{REFERENCES}

1. Sharma, and K.M. Hassan. Pathophysiologic mechanisms of acute ischemic stroke: An overview with emphasis on therapeutic significance beyond thrombolysis. Pathophysiology. 2010;17(3):197-18.

2. Khatib A and R Jamal. The impact of inpatient collaborative clinical pharmacy renal dosing service on dosage adjustment in patients with chronic kidney disease [RS1-441]. Universiti Sains Malaysia; 2009.

3. Evans KJ and A Greenberg. Hyperkalemia: a review. Journal of Intensive Care Medicine. 2005;20(5):272-290.
4. Wargo KA, K Chong, and EC Chan. Acute renal failure secondary to angiotensin II receptor blockade in a patient with bilateral renal artery stenosis. Pharmacotherapy: The Journal of Human Pharmacology and Drug Therapy. 2003;23(9):1199-204.

5. Adams $\mathrm{H}$, et al. Guidelines for the Early Management of Patients With Ischemic Stroke 2005 Guidelines Update A Scientific Statement From the Stroke Council of the American Heart Association/American Stroke Association. Stroke. 2005;36(4):916-23.

6. Wood AJ, T Brott and J Bogousslavsky. Treatment of acute ischemic stroke. New England Journal of Medicine. 2000;343(10):710-722. 